\title{
UAV Altitude Estimation by Mixed Stereoscopic Vision
}

\author{
Damien Eynard and Pascal Vasseur and Cédric Demonceaux and Vincent Frémont
}

\begin{abstract}
Altitude is one of the most important parameters to be known for an Unmanned Aerial Vehicle (UAV) especially during critical maneuvers such as landing or steady flight. In this paper, we present mixed stereoscopic vision system made of a fish-eye camera and a perspective camera for altitude estimation. Contrary to classical stereoscopic systems based on feature matching, we propose a plane sweeping approach in order to estimate the altitude and consequently to detect the ground plane. Since there exists a homography between the two views and the sensor being calibrated and the attitude estimated by the fish-eye camera, the algorithm consists then in searching the altitude which verifies this homography. We show that this approach is robust and accurate, and a CPU implementation allows a real time estimation. Experimental results on real sequences of a small UAV demonstrate the effectiveness of the approach.
\end{abstract}

\section{INTRODUCTION}

Unmanned Aerial Vehicles (UAVs) have received a lot of attention in the last decade in order to increase their autonomy. This autonomy includes the capacity of performing maneuvers such as landing, takeoff or steady flight. Thus, a fast and accurate estimation of parameters such as altitude, attitude and velocities are required by the control loop. In this paper, we propose a new mixed fish-eye/perspective stereoscopic vision system which is able to estimate autonomously the altitude of the UAV but also to provide its attitude and the free ground plane areas.

Altitude can be obtained by different techniques using Global Positioning System (GPS), altimeter (laser or pressure), radar or computer vision. However, standard GPS have a vertical precision between 25 meters and 50 meters and are sensitive to transmission interruptions in urban environment for example. For pressure altimeters, the main drawback is the dependance to pressure variation which implies an accuracy error between $6 \%$ and $7 \%$. Laser altimeters are very accurate but require specific conditions about the reflection surface. Finally, radar sensors provide simultaneously altitude and relief map but are active systems, possibly detectable and energy consuming.

Computer vision techniques have been also increasingly used during the last decade in order to estimate UAV parameters and many systems have been proposed to measure the altitude. Altitude estimation by vision systems presents several advantages. First, it can be also used for other visual tasks like obstacle avoidance, navigation or localization.

This work is supported by Région Picardie Project ALTO

D. Eynard, P. Vasseur and C. Demonceaux are with MIS Lab of University of Picardie Jules Verne, Amiens, France. firstname.namedu-picardie.fr

V. Frémont and P. Vasseur are with Heudiasyc Lab of University of Technology of Compiègne, France. vincent. fremontehds. utc. fr
Next, cameras are passive systems which are low energy consumers and which can provide a great amount of information per second. The main difficulty in systems based on vision consists in selecting an appropriate reference to estimate the altitude. In [14], [28], [29], the authors propose to use a downward-looking perspective camera in order to estimate the altitude according to a predefined pattern fixed on the ground. This kind of approach is interesting since it requires a single camera, provides a complete pose and can be used in real-time. Nevertheless it is limited to specific environment equipped with artificial landmarks. A single perspective camera has been also used in many systems based on optical flow measurement [1], [4], [6] and [16]. These systems are inspired by bees and consist in deducing the altitude according to the optical flow knowing the speed of the camera. [4] proposes to also estimate the pitch in order to correct the optical flow contrary to the others, which may lead to an unstable system. An original work using a single perspective camera has been also proposed in [7]. The authors use a technique based on the learning of the mapping between the texture information contained in a topdown aerial image to a possible altitude value. This learning is made for different kinds of ground and a spatio-temporal Markov Random Field. In [27], a multi view algorithm based on the sequence obtained with a single camera is proposed in order to compute a digital map of the ground. Rather than using a single camera which may lead to an insufficient amount of features between successive images, some authors propose to use stereoscopic sensors [20][26]. The proposed approaches are based on the matching of interest points in order to deduce elevation maps of the ground.

In this paper, we also propose the use of an original stereoscopic sensor slightly different from the previously mentionned systems since it is constituted of two different cameras respectively fisheye and perspective. The benefit of large field of view cameras for UAVs has been already demonstrated in different works such as [19] for navigation or [12] for attitude estimation. The use of a mixed sensor allows to obtain a large field of view with the fisheye lens while the perspective camera provides a better accuracy in the image. We just assume that the ground plane is dominant in the perspective image and the stereovision sensor is calibrated. In this way, there exists a homography between the two images of the ground plane. Since, we are able to estimate the attitude of the UAV by the omnidirectional image as in [12] or [11], we can deduce the normal of the ground plane and finally find the altitude which verifies this homography (fig. 2, fig.4). Then we propose a plane sweeping algorithm in order to solve this task. 


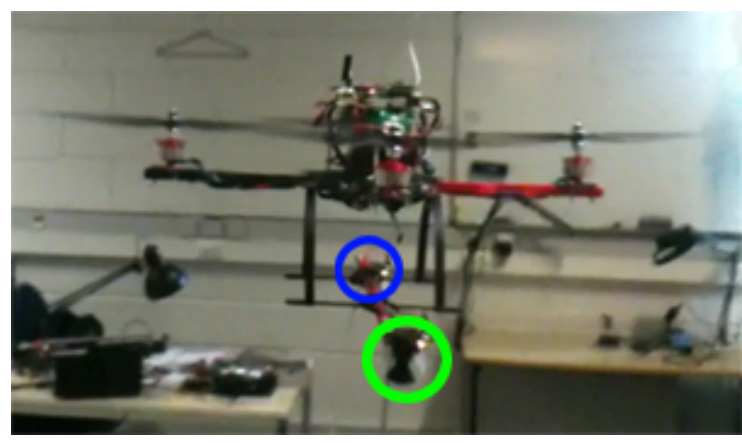

Fig. 1. Mixed system on a quadri-rotor

Briefly, our approach presents several contributions. First, the system is able to estimate autonomously the altitude without any other sensor and also provides the attitude and the ground plane area. Next, we propose a correspondencefree approach which allows to treat images with different geometry (spherical and planar) and is particularly more robust than classical matching based stereoscopic approaches. Finally, a CPU implementation allows a real time altitude estimation on small UAV.

The rest of the paper is as follows. Part II deals with the general principle with a global overview of the hybrid system, its modeling and then the plane-sweeping. In part III, we propose the plane-sweeping of mixed views to estimate the altitude and segment the ground plane. We finally present in part IV experimental results on real sequences with a quantitative evaluation of the error and a real time implementation on a small quadri-rotor UAV.

\section{GENERAL PRINCIPLE}

\section{A. Hybrid sensor}

1) Global Overview: We propose a mixed perspective/omnidirectional stereovision system (fig. 1) which is able to estimate altitude in real time as well as the ground plane and the attitude.

The advantage of the omnidirectional sensor is the wide field of view while the drawbacks are the poor resolution (particularly near the borders), non linear resolution of the image and the distortions. Advantages of fisheye in comparison with catadioptric cameras are their reduced sensitivity to vibrations and the suppression of the blind spot at the center of the image.

On the other side, perspective cameras possess a good and constant resolution, low distortions but a limited field of view.

By combining a such mixed stereo rig, we can add advantages of each sensor. The fisheye provides attitude information (fig. 5) whereas perspective view can provide motion information more precisely than on fisheye view. Then the main problem in our system consists in matching between the omnidirectional and the perspective images because of the distortions. Different approaches are then possible:

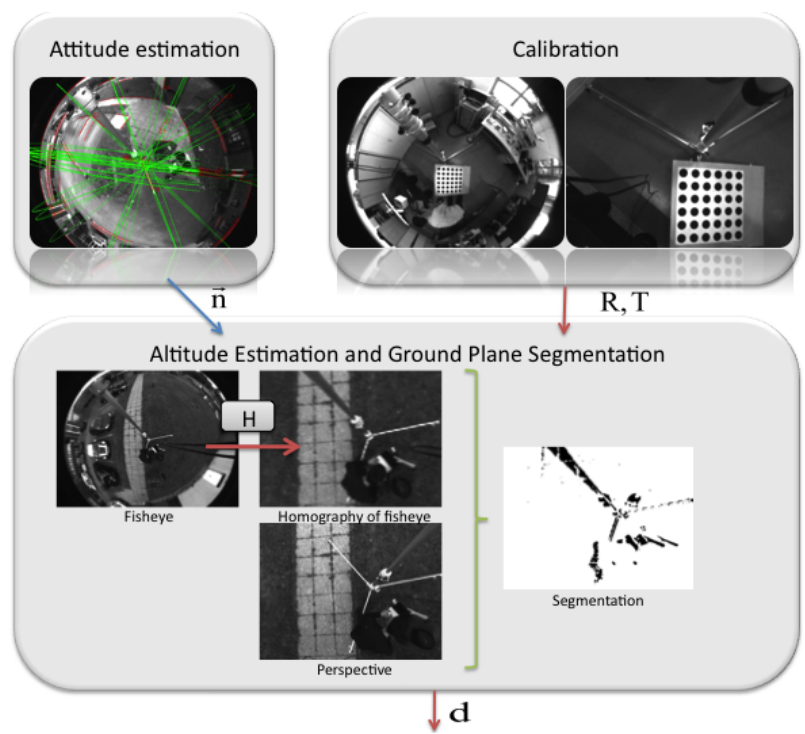

Fig. 2. Global overview of altitude estimation

- First, by knowing the intrinsic parameters of the fisheye camera, a rectified equivalent perspective image could be recovered in order to perform for example, a feature matching. However, this approach requires different processings such as warping and interpolation which decrease a real time performances.

- Some recent works propose the unitary sphere as unified space for central image processing and feature matching. However, as previously, this solution can not be implemented in real time and is not adapted for mixed view.

- Finally, we propose a real time correspond-less approach which consists in comparing directly the images without any feature extraction.

Since the altitude is estimated according to the ground plane, we can use this plane as reference. In this way, we will demonstrate that there exists a homography between the omnidirectional and perspective images. The general equation of a homography is $H=R-T \frac{n^{\prime}}{d}$, where $R$ and $T$ define the rigid transformation between the two views, $n^{\prime}$ is the normal of the plane in the first image and $d$ is the distance from the plane and the first camera. In our case, $d$ corresponds to the altitude (see fig. 4).

Consequently, if we are able to find this homography between the two images, we can deduce $d$ since $R$ and $T$ can be known by calibration and $n^{\prime}$ can be computed using attitude estimation methods based on omnidirectional vision such as [3], [10], [11] or by IMU (see fig. 2).

2) Camera Models and Calibration: Despite fisheye lenses cannot be classified as single viewpoint sensor [2], we use the unitary sphere in order to model our camera [31]. Mei and Rives [25] have proposed a calibration method based on this spherical model. This model is particularly accurate and allows to model radial and tangential distortions of the fisheye lens.

With the spherical model of [25], projection is divided 
in two steps. First, a world point $x_{m}$ is projected onto the unit sphere $x_{s}$ through its center $S$. Then, this point $x_{s}$ is projected to the image plane onto $x_{i}$ through $O$. Parameter $\xi$ defines the distance between $O$ and $S$. This parameter is estimated during the calibration. Mixed stereo calibration is obtained by an adaptation of [5].

\section{B. Plane-sweeping}

Plane-sweeping has been introduced by Collins [8]. First a reference view has to be defined. Then for each normal and each distance to a 3D plane, each warped image is compared (eq. 1) by homography to the reference image. Let $I(p)$ be the intensity of the pixel $p$ in the image $I$ and $I^{*}$ the homography of the image $I$ by the homography $H_{p}$.

$$
I^{*}(p, d)=I\left(H_{p}\right)=I\left(\left(R-T \frac{n^{\prime}}{d}\right) p\right)
$$

The best estimation of the homography $H$ corresponds to the minimum global error of the difference between the warped image and the reference view. In our application, we extend this aspect. We take perspective $I_{p}$ view as the reference. The manipulation with the neighborhood on a plane is more feasible than on a sphere. The image obtained with fisheye camera is projected on the sphere and then on the reference plane by homography. Notice our cameras have the same orientation. The region in consideration on the fisheye gets fewer distortions and better resolution than the rest of the image. Then those two images can be compared by subtraction (see fig. 3). We note $I_{p}$ the perspective image, $I_{s}$ the fisheye image projected onto the sphere and $I_{s}^{*}$ the $I_{s}$ image projected by homography onto the reference frame.

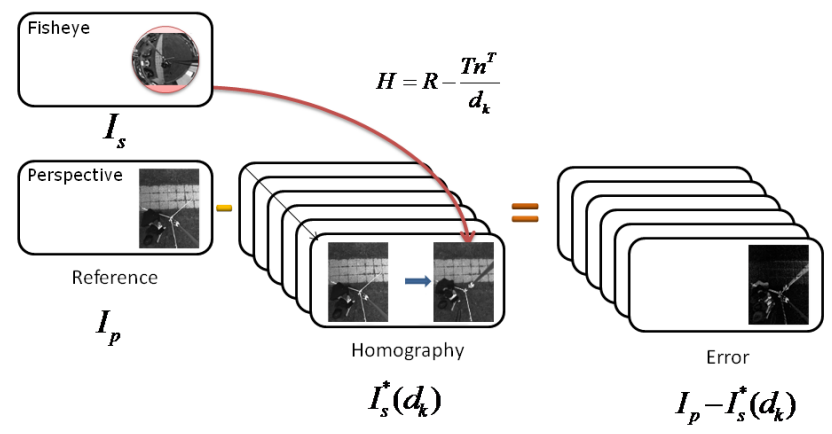

Fig. 3. Mixed plane-sweeping.

\section{PLANE-SWEEPING OF MIXED VIEWS}

We propose to estimate the altitude $d$ and to segment the ground plane by mixed plane-sweeping with $R, T, n^{\prime}$ known by calibration and attitude estimation. First of all, we will present the homography used in our models. Secondly, we will expose the plane-sweeping of mixed views algorithm.

\section{A. Sphere to plane homography}

Given a mixed stereo rig modeled by a plane and a unit sphere, we propose in this part to define the homography of the $3 \mathrm{D}$ ground plane that exists between the two views from different types of cameras (see fig. 4). In [17], a homography links two projections of a 3D plane on two planes. In [24], homography links two projections of a 3D plane on two spheres:

$$
H=R-T \frac{n^{\prime}}{d}
$$

Let us consider:

- $X_{p}$, a point of the $3 \mathrm{D}$ plane projected on the perspective view.

- $X_{p}^{*}$, the projection of $X_{p}$ from a perspective view to another perspective view by homography.

- $X_{s}$, a point of the 3D plane projected on the sphere.

We have the following relation (3) for a homography between two perspective views.

$$
X_{p}^{*} \sim H^{-1} X_{p}
$$

We replace those two planes of projection by a planar and a spherical projection. We get eq. 4 up to scale:

$$
X_{s} \sim \frac{X_{p}^{*}}{\left\|X_{p}^{*}\right\|} \sim H^{-1} X_{p}
$$

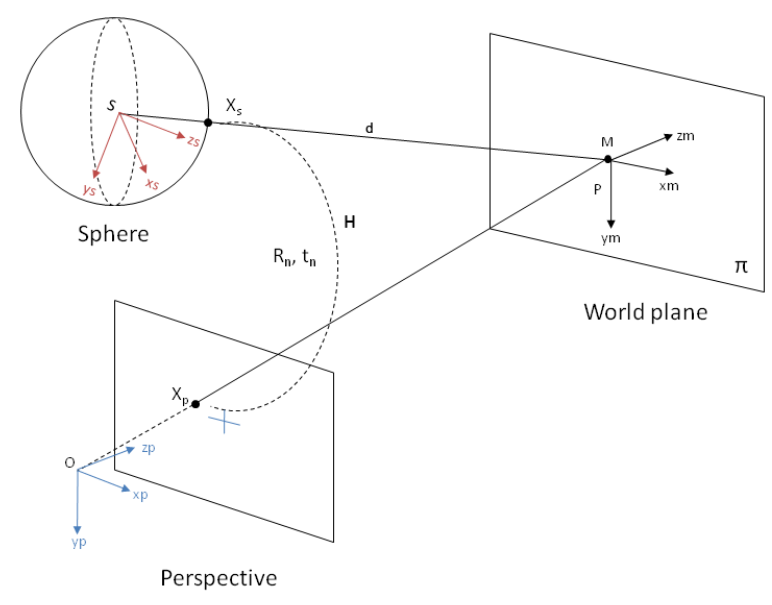

Fig. 4. Sphere/plane homography.

As regard what we have said previously, homography $H$ depends on $R, T$, attitude $\vec{n}$ and altitude $d$. Rotation $R$ and translation $T$ are obtained by calibration. Normal $\vec{n}$ to the ground plane can be obtained by [3], [10], [11] or by inertial system. [3] work has been tested on a fisheye view (fig. 5). Finally, we will estimate the altitude $d$ by plane-sweeping.

\section{B. Algorithm}

To estimate the altitude parameter, our plane-sweeping algorithm performs a top-down search of the altitude. Let $d_{\min }$ and $d_{\max }$ be the minimum and maximum altitude to estimate. In each iteration, the best altitude $\widehat{d_{k}}$ is estimated from a range $d_{k} \epsilon\left[d_{\min }, d_{\max }\right]$, then the mask $G$ is updated (see algorithm 1). In this algorithm, the mask $G$ corresponds to the segmented ground plane. Pixels corresponding to the ground plane are in white color in figures 6(c). In order to obtain a real time method, we propose to estimate the altitude 


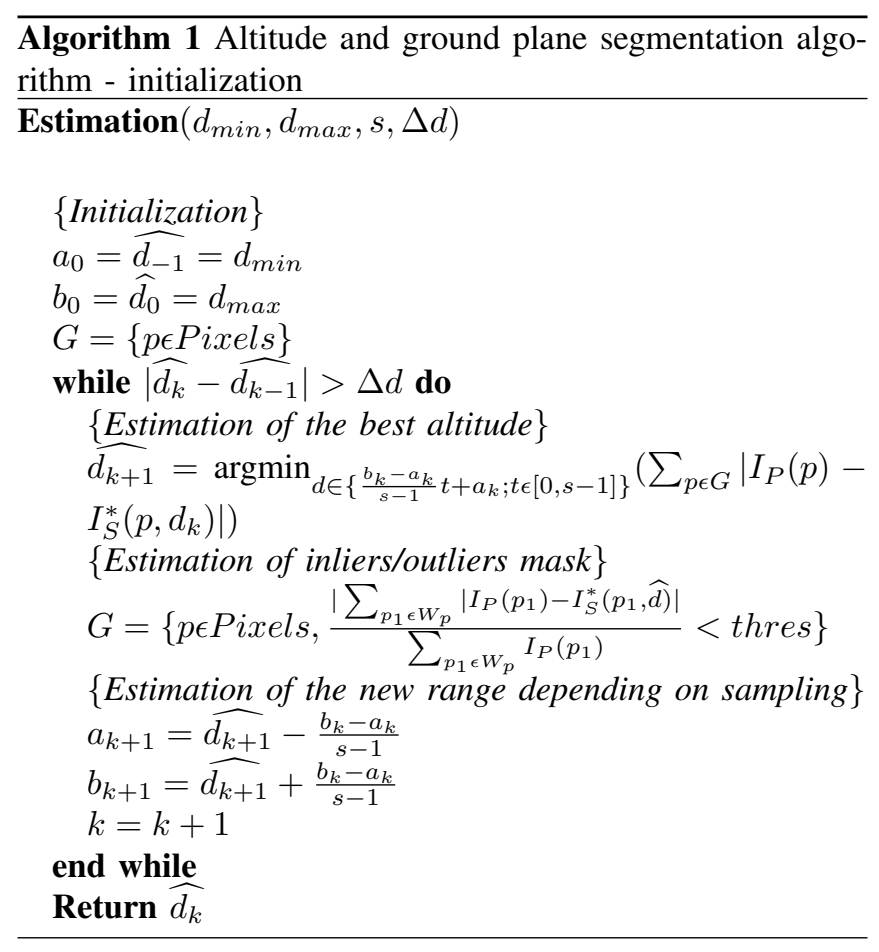

at time $t$ using time $t-1$. We define $\Delta d$ the tolerance of altitude and $s$ the step.

The estimation is performed in two phases:

- Initialization: we estimate the best altitude which is in a wide range of altitudes (algorithm 1).

- During flight: we use the altitude estimated in the initialization phase to obtain a narrower range $d_{t} \epsilon\left[d_{t-1}-\right.$ $\left.r_{d}, d_{t-1}+r_{d}\right]$ by substituting in (algo. 1) $d_{\text {min }}=$ $d_{t-1}-r_{d}, d_{\max }=d_{t-1}+r_{d}$ with $r_{d}$ computed in (eq. 5,6). This range depends on the vertical velocity $v_{v}$ of UAV (about $\pm 5000 \mathrm{~mm} / \mathrm{s}$ ) and the hardware computation power in frames per second noted $f p s$.

$$
\begin{gathered}
r_{d}=\frac{v_{v}}{f p s} \\
d_{t} \epsilon\left[d_{t-1}-r_{d}, d_{t-1}+r_{d}\right]
\end{gathered}
$$

As we will see in the following section, our algorithm is able to compute both the altitude and the ground plane in real time with mixed stereo system.

\section{EXPERIMENTAL RESULTS}

We can distinguish our results in two parts: firstly, images are processed offline, with standard cameras like Sony XCDV50CR. In the second part, images are processed online for embedded applications with micro-cameras with M12 fisheye and perspective lens. In each experiment, we estimate the attitude with an inertial central (IMU) to validate our approach.

\section{A. Attitude estimation by fisheye lens}

For the attitude estimation, we get results from an IMU to have the less error and the best computation time. In the

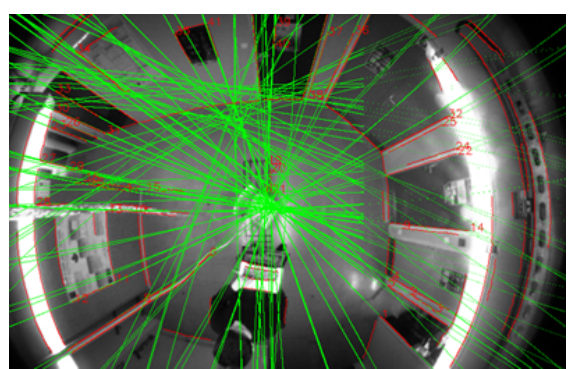

Fig. 5. Error of attitude estimation $=0.69^{\circ}$ - red lines are detected edges, green lines are 3D lines projected in the image

future, we will use an adaptation of [12] and [3]. The error introduced with this method has a maximum of $3^{\circ}$. Our algorithm is insensitive to low attitude errors. For example, with synthetic images, an error between $3^{\circ}$ and $5^{\circ}$ of attitude estimation will introduce an error between $0.1 \%$ and $0.4 \%$ for altitude estimation. This method tested on our fisheye lens estimates the attitude as well as catadioptric lens does (see fig. 5).

\section{B. Altitude estimation and/or ground plane segmentation}

Then, we present two cases of experimental results where real altitude is estimated by a laser telemeter and error computed like error $=\frac{\text { (estim_altitude-real_altitude) }}{\text { real_altitude }}$.

- the first, made with two cameras with a $447 \mathrm{~mm}$ baseline. It is fixed on a pneumatic telescopic mast. Altitude and ground plane estimation are performed offline on a GPU.

- the second, made with two micro cameras with a $314 \mathrm{~mm}$ baseline. It is embedded on a compact UAV. Altitude estimation is performed online by CPU processing.

For the first experiment, in the one hand, we observe an accurate estimation of altitude on free ground plane (tab. I) with an error between $0.18 \%$ and $3.14 \%$ in case of a free ground plane. In case of obstacles on the ground plane, we observe a higher error, between $7.52 \%$ and $8.82 \%$. In the other hand we observe that higher is our system, less accurate is the estimation because of the decrease of resolution in function of altitude. Moreover, the accuracy depends on the size of the baseline. Finally those results are well adapted for our application. Accuracy is needed during the two phases of landing and taking off i.e. near to the ground plane.

\begin{tabular}{|c|c|c|c|}
\hline Type & Ground truth & Estim. altitude & Error \\
\hline Ground & $2187 \mathrm{~mm}$ & $2200 \mathrm{~mm}$ & $0.59 \%$ \\
\hline Ground & $3244 \mathrm{~mm}$ & $3250 \mathrm{~mm}$ & $0.18 \%$ \\
\hline $\begin{array}{c}\text { Ground + obstacles } \\
\text { (low contrast) }\end{array}$ & $3244 \mathrm{~mm}$ & $3488 \mathrm{~mm}$ & $7.52 \%$ \\
\hline Ground & $4072 \mathrm{~mm}$ & $4200 \mathrm{~mm}$ & $3.14 \%$ \\
\hline Ground & $5076 \mathrm{~mm}$ & $5202 \mathrm{~mm}$ & $2.48 \%$ \\
\hline Ground + obstacles & $4080 \mathrm{~mm}$ & $4440 \mathrm{~mm}$ & $8.82 \%$ \\
\hline
\end{tabular}

TABLE I

ALtitude GROUND PLANE ESTIMATION WITH AND WiTHOUT OBSTACLE - Algorithm PARAMETERS FOR THIS TEST: $s=6$, thres $=25$ 
The second aspect of our algorithm is the segmentation of ground plane, well estimated for contrasted areas. In case of a plane without obstacles, the pneumatic telescopic mast, where cameras are fixed, is well represented by outliers (in dark on the image) (fig. 6(c)). For an image composed of a dominant ground plane and walls, ground plane is segmented as inliers while walls are segmented as outliers. The detected area of inliers is $31 \%$ while an accurate estimation of inliers area gives $54 \%$. Our algorithm allows to segment globally inliers/outliers to estimate dominant ground plane for our application.

An aspect to improve in our algorithm is the case of poorly textured planes. When the ground plane or outliers (wall, objects) are homogeneous or poorly textured, outliers/inliers segmentation becomes difficult.

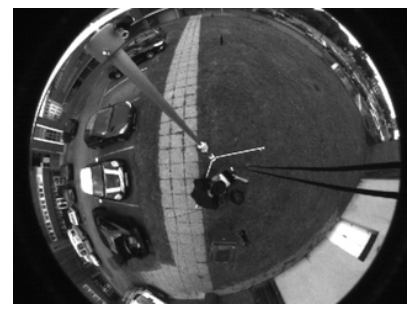

(a)

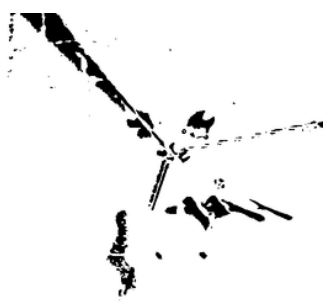

(c)

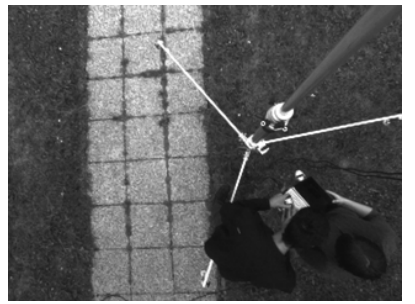

(b)

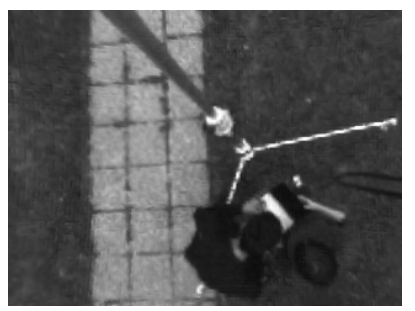

(d)

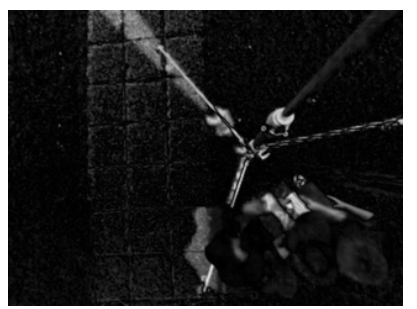

(e)

Fig. 6. Altitude and ground plane segmentation - $4.8 \%$ of inliers - Fisheye view (a), perspective view (b), ground plane segmentation (c), sphere to plane homography (d), reference and homography comparison (e)

For the second experiment we implemented our system on a small quadri-rotor (see fig.1). Micro cameras embedded on the UAV (see fig. 7) are plugged on external laptop to perform online altitude estimation. We tested the accuracy by comparing altitudes estimated by planesweeping to altitudes estimated by laser telemeter (Fig.8). On this figure, altitude is well estimated for the range of altitude corresponding to the landing and taking off phases of an UAV. The mean error is $2.41 \%$. An attached video shows an example of this experiment and others are available [35].

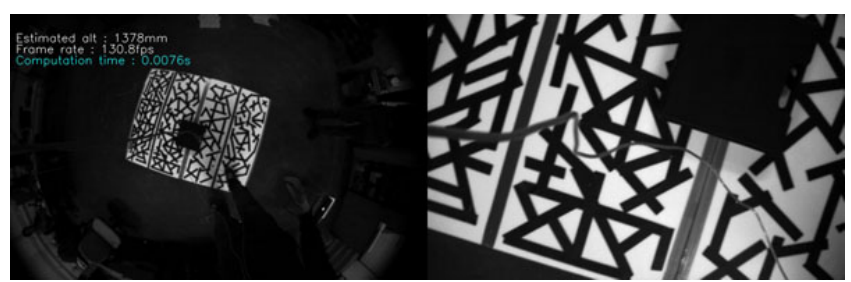

Fig. 7. Embedded view of UAV - Est. altitude 1378mm

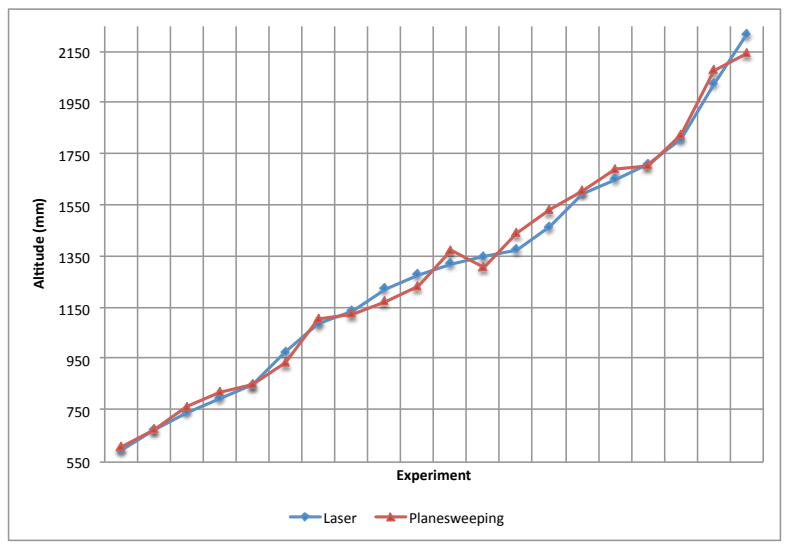

Fig. 8. Comparison between laser altimeter and planesweeping

\section{Performance on GPU, CPU and embedded boards}

First, we developed this algorithm on GPU with brook+ for ATI that allows to get real time $(30 \mathrm{~Hz})$ frame-rate to estimate both altitude and segment the ground plane. This algorithm has been tested on ATI 4850 with E8400 3Ghz CPU.

Then, we implemented this algorithm on CPU with a lot of optimizations and without the segmentation of the ground plane. With this implementation, we get min : $80 \mathrm{~Hz}$, mean : $180 \mathrm{~Hz}, \max : 250 \mathrm{~Hz}$ that is above video framerate which allows us to process our algorithm online. The platform for those tests is a Macbook Pro with a CPU C2D P8400 2.26Ghz. A demonstration has been developed [33]. We use a stereo rig with uEye cameras and get the normal with an IMU. During this demonstration, the system is able to estimate altitude in real time with robustness and accuracy.

An embedded version of our algorithm has been exported on the ARM of a Gumstix Overo Fire with OMAP3530 ARM @ 600Mhz based processor. With this implementation we get a framerate around $5 \mathrm{~Hz}$ that is not enough for real time applications but relatively interesting for the ratio power/size.

By developing those algorithms both on GPU, CPU and embedded board we get interesting results. For ground plane estimation and altitude estimated together, results are real time and can be implemented on an UAV with GPU. For altitude estimation only, computation time is faster and can be implemented on smaller quadri-rotor UAV.

We implemented and validated the altitude estimation by CPU on a light quadri-rotor [33]. Algorithm has been performed on a macbook pro in real-time and during the 
flight.

\section{CONCLUSIONS AND FUTURE WORKS}

We have presented in this paper a hybrid stereo system. Mixed cameras are related by a homography which allows to estimate both altitude and ground plane using plane-sweeping. Compared to matching algorithms based on feature matching, plane-sweeping is a correspondence-free algorithm. It consists of directly comparing images. This algorithm tests a range of altitudes and extracts the best one where the global error is minimum. First, we have implemented this algorithm on GPU and have presented good preliminary results in video [34]. Then, we implemented the algorithm of altitude estimation on CPU. A version of laptop used for demonstration has a framerate around $180 \mathrm{~Hz}$ and has been implemented on a real UAV. A second version has been developed on an embedded board which has a size of stick of gum with a framerate of $5 \mathrm{~Hz}$. Notice on the video a bigger computation time due to the processing of the video realized during the flight.

Perspectives of this work will be to improve our algorithm for the segmentation on poorly textured surfaces and to implement an onboard version of our approach.

\section{ACKNOWLEDGMENTS}

This work is supported by Région Picardie Project ALTO. Experimentations have been realized on UAV platform of Heudiasyc with cooperation of Luis-Rodolfo GARCIACARRILLO and Eduardo RONDON. A mixed calibration software has been developed with cooperation of Guillaume CARON.

\section{REFERENCES}

[1] G. Barrows and C. Neely and K. Miller, "Optic flow sensors for MAV navigation," In Fixed and Flapping Wing Aerodynamics for Micro Air Vehicle Applications, ser. Progress in Astronautics and Aeronautics, T. J. Mueller, Ed. AIAA, 2001, vol. 195, pp. 557-574.

[2] S. Baker, and S. K. Nayar, "A Theory of Single-Viewpoint Catadioptric Image Formation," International Journal of Computer Vision,1999.

[3] J-C Bazin, I. Kweon, C. Demonceaux, P. Vasseur, "UAV Attitude Estimation by Vanishing Points in Catadioptric Images", In Proceedings of IEEE International Conference on Robotics and Automation, 2008.

[4] A. Beyeler and C. Mattiussi and J.C. Zufferey and D. Floreano, "Vision-based Altitude and Pitch Estimation for Ultra-light Indoor Microflyers," In Proceedings of IEEE International Conference on Robotics and Automation, 2006.

[5] G. Caron, E. Marchand, E. Mouaddib. "Single Viewpoint Stereoscopic Sensor Calibration", In Int. Symp. on Image/Video Communications over fixed and mobile networks, 2010.

[6] J. Chahl and M. Srinivasan and and H. Zhang, "Landing strategies in honeybees and applications to uninhabited airborne vehicles," In The International Journal of Robotics Research, vol. 23, no. 2, pp. 101-110, 2004

[7] A. Cherian and J. Andersh and V. Morellas and N. Papanikolopoulos and B. Mettler, "Autonomous Altitude Estimation Of A UAV Using A Single Onboard Camera," In Proceedings of IEEE International Conference on Intelligent and Robotic Systems, 2009.

[8] R. T. Collins, "A space-sweep approach to true multi-image matching," In Proceedings of IEEE Computer Vision and Pattern Recognition, 1996.

[9] J. Courbon, Y. Mezouar, L. Eck, P. Martinet, "A Generic Fisheye camera model," In Proceedings of IEEE International Conference on Intelligent and Robotic Systems, 2007.
[10] C. Demonceaux, P. Vasseur, C. Pégard, "Robust Attitude Estimation with Catadioptric Vision," In IEEE/RSJ International Conference on Intelligent Robots and Systems, 2006.

[11] C. Demonceaux and P. Vasseur and C. Pégard, "Omnidirectional vision on UAV for attitude computation," In Proceedings of IEEE International Conference on Robotics and Automation, 2006.

[12] C. Demonceaux and P. Vasseur and C. Pégard, "UAV Attitude Computation by Omnidirectional Vision in Urban Environment," In Proceedings of IEEE International Conference on Robotics and Automation, 2007.

[13] D. Gallup, J.-M. Frahm, P. Mordohai, Y. Qingxiong and M. Pollefeys, "Real-Time Plane-Sweeping Stereo with Multiple Sweeping Directions," In Proceedings of IEEE Computer Vision and Pattern Recognition, 2007.

[14] P. Garcia-Padro and G. Sukhatme and J. Montgomery, "Towards visionbased safe landing for an autonomous helicopter," In Robotics and Autonomous Systems, 2000.

[15] I. Geys, T. P. Koninckx, L. V. Gool, "Fast Interpolated Cameras by combining a GPU based Plane Sweep with a Max-Flow Regularisation Algorithm," In Proceedings of IEEE 3D Processing, Visualization and Transmission, 2004.

[16] W. Green and P. Oh and K. Sevcik and G. Barrows, "Autonomous landing for indoor flying robots using optic flow," in ASME International Mechanical Engineering Congress and Exposition, vol. 2, 2003 , pp. 1347-1352.

[17] R. Hartley and A. Zisserman, "Multiple View Geometry in computer vision," Cambridge 2cnd edition, 2003.

[18] J. Hoffmann, M. Jungel and M. Lotzsch, "Vision Based System for Goal-Directed Obstacle Avoidance," In 8th International Workshop on RoboCup, 2004.

[19] S. Hrabar and G. Sukhatme, "Omnidirectional vision for an autonomous helicopter," In Proceedings of IEEE International Conference on Robotics and Automation, 2004.

[20] I.K. Jung and S. Lacroix, "High resolution terrain mapping using low altitude aerial stereo imagery," In Proceedings of IEEE International Conference on Computer Vision, 2003.

[21] Y. Kim and H. Kim, "Layered ground floor detection for visionbased mobile robot navigation," In Proceedings IEEE International Conference on Robotics and Automation, 2004.

[22] B. Liang and N. Pears "Visual Navigation using Planar Homographies," In Proceedings of IEEE International Conference on Robotics and Automation, 2002.

[23] Y. Ma, S. Soatto, J. Kosecka, ans S. Shankar Sastry, "An invitation to 3D Vision," Springer, 2003.

[24] C. Mei, S. Benhimane, E. Malis and P. Rives, "Homography-based Tracking for Central Catadioptric Cameras," In Proceedings of IEEE Intelligent Robots and Systems, 2006.

[25] C. Mei and P. Rives, "Single View Point Omnidirectional Camera Calibration from Planar Grids," In Proceedings of IEEE International Conference on Robotics and Automation, 2007

[26] M. Meingast and C. Geyer and Shankar Sastry, "Vision Based Terrain Recovery for Landing Unmanned Aerial Vehicles," In Proceedings of IEEE International Conference on Decision and Control, 2004.

[27] M. Sanfourche and G. Besnerais and S. Foliguet, "Height estimation using aerial side looking image sequences," In ISPRS, Vol.XXXIV, Part3/W8, Munich, 2003.

[28] S. Saripalli and J. Montgomery and G. Sukhatme, "Vision based autonomous landing of an unmanned aerial vehicle," In Proceedings of IEEE International Conference on Robotics and Automation, 2002.

[29] C. Sharp and O. Shakernia, and S. Sastry, "A vision system for landing an unmanned aerial vehicle," In Proceedings of IEEE International Conference on Robotics and Automation, 2001.

[30] P. Sturm, "Mixing Catadioptric and Perspective Cameras," In Proceedings of IEEE Omnidirectional Vision,2002

[31] X. Ying and Z. Hu, "Can We Consider Central Catadioptric Cameras and Fisheye Cameras within a Unified Imaging Model," In European Conference on Computer Vision, 2004.

[32] J. Zhou and B. Li, "Robust Ground Plane Detection with Normalized Sequences from a Robot Platform," In Proceedings of IEEE International Conference on Image Processing, 2006

[33] http://www.youtube.com/watch?v=NqGL8h9zGd0, 2010

[34] http://www.youtube.com/watch?v=UjiT3q9VN1g, 2010

[35] http://www.youtube.com/watch?v=ubXzf0eLud4, 2010 